\title{
Prognostic implications of trunk muscle mass in liver cirrhosis
}

\author{
Jimin Han and Won Kim \\ Division of Gastroenterology and Hepatology, Department of Internal Medicine, Seoul Metropolitan Government Seoul National \\ University Boramae Medical Center, Seoul National University College of Medicine, Seoul, Korea
}

Keywords: Sarcopenia; Cirrhosis; Prognosis; Psoas muscle

\section{See Article on Page 319}

In 1988, Rosenberg suggested that sarcopenia is the age-associated decline of lean body mass. ${ }^{1}$ However, the European Working Group on Sarcopenia in Older Persons defined sarcopenia as a syndrome characterized by a progressive and generalized loss of skeletal muscle mass with either muscle weakness or poor physical performance, since the relationship between muscle strength and muscle mass is not linear. ${ }^{2}$ Sarcopenia can be considered 'primary' when no other cause but ageing itself is evident, while sarcopenia can be considered 'secondary' when one or more other causes, such as inflammatory disease, malignancy, endocrine disease, and advanced organ failure, are evident. ${ }^{2}$

The prevalence of sarcopenia among patients with liver cirrhosis was reported to be average $48.1 \%{ }^{3}$ Sarcopenia is associated with poor prognosis including higher risk of mortality in patients with cirrhosis. There are multiple factors which may contribute to sarcopenia in cirrhosis. The causes responsible for sarcopenia in pa- tients with liver cirrhosis include malnutrition, elevated myostatin level, abnormal use of protein as an energy source, activation of the inflammatory cytokines, and hormone deficiency. ${ }^{4}$

There are various methods in clinical use for diagnosing low muscle mass. In the past, urine creatinine was used as an indicator of human muscle mass. Other diagnostic methods include anthropometric assessments, bioelectrical impedance analysis, and plethysmography. However, due to the recent advance of radiological examinations, dual energy $\mathrm{X}$-ray absorptiometry, computed tomography $(\mathrm{CT})$, and magnetic resonance imaging have been mainly used to measure muscle mass for practical or investigational purpose. ${ }^{5}$

In the current issue, Gu et al. evaluated the optimal cutoff values of psoas muscle thickness per height (PMTH) by gender for detecting sarcopenia in cirrhotic patients. ${ }^{6}$ The skeletal muscle index (SMI), as assessed by the cross-sectional area of several muscles at the $\mathrm{L} 3$ vertebral level on CT, is one of the most widely used techniques. Moreover, PMTH was well correlated with SMI in their work. SMI-sarcopenia and sex-nonspecific cutoff of PMTH-sarco-

\section{Abbreviations:}

CT, computed tomography; PMTH, psoas muscle thickness per height; SMI, skeletal muscle index

\section{Corresponding author: Won Kim}

Division of Gastroenterology and Hepatology, Department of Internal Medicine, Seoul Metropolitan Government Seoul National University Boramae Medical Center, Seoul National University College of Medicine, 20 Boramae-ro 5-gil, Dongjak-gu, Seoul 07061, Korea

Tel: +82-2-870-2233, Fax: +82-2-831-2826

E-mail:drwon1@snu.ac.kr

https://orcid.org/0000-0002-2926-1007

Received : Aug. 9, 2018/ Accepted : Aug. 16, 2018

Copyright $\odot 2018$ by Korean Association for the Study of the Live

This is an Open Access article distributed under the terms of the Creative Commons Attribution Non-Commercial License (http://creativecommons.org/licenses/by-nc/3.0/) which permits unrestricted non-commercial use, distribution, and reproduction in any medium, provided the original work is properly cited. 
penia were significantly associated with mortality in patients with cirrhosis, but there was no significant association between sexspecific cutoffs of PMTH sarcopenia and mortality. The current work also adds to the growing body of literature investigating the prognostic role of sarcopenia in patients with liver cirrhosis.

Although there is a substantial limitation that PMTH cannot be measured at precise locations in patients with ascites or osteoporosis disease, the current study provides a simple and valuable criterion for measuring muscle mass with $\mathrm{CT}$ scan in patients with liver cirrhosis. In addition, more complete diagnostic criteria would be established if they further validate the diagnostic criteria of sarcopenia according to various etiologies of cirrhosis as well as gender of patients with liver cirrhosis.

\section{Authors' contribution}

Manuscript writing: Jimin Han, Won Kim

Final approval of manuscript: Won Kim

\section{Conflicts of Interest}

The author have no conflicts to disclose.

\section{REFERENCES}

1. Rosenberg IH, Roubenoff R. Stalking sarcopenia. Ann Intern Med 1995;123:727-728.

2. Cruz-Jentoft AJ, Baeyens JP, Bauer JM, Boirie Y, Cederholm T, Landi $F$, et al. Sarcopenia: European consensus on definition and diagnosis: report of the European Working Group on Sarcopenia in Older People. Age Ageing 2010;39:412-423.

3. Kim G, Kang SH, Kim MY, Baik SK. Prognostic value of sarcopenia in patients with liver cirrhosis: a systematic review and meta-analysis. PLoS One 2017;12:e0186990.

4. Sinclair M, Gow PJ, Grossmann M, Angus PW. Review article: sarcopenia in cirrhosis--etiology, implications and potential therapeutic interventions. Aliment Pharmacol Ther 2016;43:765-777.

5. Cooper C, Fielding R, Visser M, van Loon LJ, Rolland $Y$, Orwoll $E$, et al. Tools in the assessment of sarcopenia. Calcif Tissue Int 2013;93:201-210.

6. Gu DH, Kim MY, Seo YS, Kim SG, Lee HA, Kim TH, et al. Clinical usefulness of psoas muscle thickness for the diagnosis of sarcopenia in patients with liver cirrhosis. Clin Mol Hepatol 2018;24:319-330. 